PoS $\quad \begin{aligned} & \text { PROCEEDINGS } \\ & \text { OF SCIENCE }\end{aligned}$

\title{
Brane Worlds Working Group Summary
}

\section{Dileep Jatkar*}

Harish-Chandra Research Institute, Allahabad

E-mail: dileep@mri.ernet.in

This is a summary of the Brane Worlds working group at From Strings to LHC conference.

From Strings to $\mathrm{LHC}$

January 2-10, 2007

Goa, India

* Speaker. 


\section{Introduction}

The Large Hadron Collider(LHC) will be operational shortly. Apart from finding the Higgs particle to put the Standard model on firm footing we also expect LHC to give us leads to finding physics beyond the Standard model. There are several avenues beyond the Standard model that have been explored on the theoretical side. These include supersymmetry, grand unified theories, brane-world models, etc. In the workshop, 'From Strings to LHC' several of these topics were discussed in different working groups.

The brane worlds working group activity in the conference was held from January 6-9, 2007. Summary talk of the group activity was was organized on January 10, 2007.

The brane worlds working group arranged 8 talks during the activity. This was in addition to the discussions between smaller groups which were more focussed towards identifying problems and at times doing some preliminary computations.

The working group had overlapping interests with other groups like supersymmetry breaking working group as well as with the electroweak symmetry breaking working group. Due to this some of the talks belonging to the brane worlds working group were organized in the other working group activities.

The talks in the working group can be categorized into four sets.

1. Warped models

2. String cosmology

3. Moduli Stabilization

4. Phenomenology

\section{Warped Models}

Randall-Sundrum type models were reviewed earlier in the conference. In the working group, T. Gherghetta talked about Randall Sundrum set up with two 3-branes. The scenarios discussed included, Higgs boson as a pseudo-Goldstone bosons and a model of partially supersymmetric standard model. Higgs field, in this scenario is localized on the TeV scale brane. The Standard Model fields on the other hand live in the five dimensional bulk. Hierarchical nature of Yukawa couplings is attributed to the fact that the fermion wavefunctions are peaked at different locations in the bulk and have different overlap with the TeV brane. Smaller overlap with the TeV brane leads to smaller value for the Yukawa coupling and larger overlap gives larger Yukawa coupling. The $\mathrm{TeV}$ brane localized Higgs field can be thought of as a pseudo-Goldstone coming from the $S O(5)$ gauge symmetry of the bulk theory. The $S O(5)$ gauge symmetry appears as a global symmetry in the boundary,i.e., brane theory. At the boundary this symmetry is broken to $S O(4)$. This symmetry breaking gives rise to 4 Pseudo-Goldstone bosons. These are identified as the Higgs field on the $\mathrm{TeV}$ brane. This field acquires mass due to radiative corrections coming from the bulk Standard model fields. 
Partially supersymmetric Standard Model has similar structure, i.e., the Standard Model fields are living in the bulk and the Higgs is localized on the TeV brane. The difference is that the Standard Model fields and the Higgs field belong to the supersymmetric sector on the TeV brane. Since Standard Model fields live in the bulk, the bulk theory is also supersymmetric. The supersymmetry, however, is broken on the Planck scale brane by a choice of boundary conditions. This supersymmetry breaking is then communicated to the $\mathrm{TeV}$ scale brane by radiative effects. The radiative mass for the Higgs can be shown to be less that $120 \mathrm{GeV}$. The Higgsino is a potential dark matter candidate. These scenarios give interesting alternatives to the minimal supersymmetric Standard Model.

Another talk in this category was by N. Sakai[1] on effective lagrangians on Domain walls and other solitons. Here one starts with super-Yang-Mills-Higgs theory either in five or in six dimensions and looks for a domain wall or vortex solution. These solitons have four dimensional worldvolume. The original model has $\mathscr{N}=2 U\left(N_{c}\right)$ gauge symmetry with $N_{f} \geq N_{c}$ matter multiplets. The soliton solutions are stable by virtue of being $1 / 2$ BPS solutions to the equations of motion. Using the collective coordinates method, it is possible to write down a supersymmetric $\sigma$-model for the slowly moving degrees of freedom. Interesting feature of this $\sigma$-model is that the Kähler potential of it can be written down without explicit knowledge of the metric.

\section{String Cosmology}

In this session, there were two talks. First S. Panda gave a brief survey of the string/brane cosmology. He first reviewed the cosmological Standard model and inflation using single field as well as hybrid inflation. Within the context of string theory, earlier attempts like the BrandenbergerVafa model of no initial singularity as well as the scale factor duality, i.e., $a(t) \rightarrow 1 / a(t)$ and pre-big bang inflation were reviewed. In the post D-brane era, tachyon cosmology was discussed. Models with the Dirac-Born-Infeld action multiplied by the potential energy functional for the tachyon field are novel candidates for models of inflation. These models, however, do not seem to give adequate number of e-foldings for them to be viable models of inflation. In addition to this, these models generically suffer from the $\eta$-problem. These two problems are intertwined in the slow roll inflation models. The $\eta$ parameter is a measure of slow roll of the inflaton. For slow roll inflation, $\eta \ll 1$ is necessary but in most of the tachyon inflation models, one ends up with $\eta \geq 1$. Leading to the break down of the assumption that we can have slow roll inflation in these models.

In the second talk, J. Cline[2] discussed brane-anti brane inflation models. General message about the brane-anti brane inflation models was they are quite difficult to construct given the current constraints coming from the cosmological data. He started with a quick recap of earlier attempts of brane-anti brane models. Generic models of this class also suffer from the $\eta$-problem and in this sense these models are similar to the tachyon cosmology models. There are several attempts to resolve this $\eta$-problem. For example one can add fluxes to the brane-anti brane system to give it partial stability leading to slow roll of the inflaton field. However, fluxes do not resolve the problem. One can add more terms to the inflaton potential, for example, by adding D7 branes in the Klebanov-Strassler model, but these new terms also do not alleviate the problem. 


\section{Moduli Stabilization}

The issues involving supersymmetry breaking, moduli stabilization and their implications for signals at LHC was discussed by K. Choi[3]. He pointed out that for LHC phenomenology, determining mechanism of supersymmetry breaking is not as important as how the breaking is communicated to the supersymmetric standard model sector. It is the latter which would be tested better at LHC than the mechanism itself. The requirement from the supersymmetric standard model is that the supersymmetry breaking mechanism should preserve CP and flavor at low energy. Apart from the usual mechanism of supersymmetry breaking like, minimal supergravity, gauge mediated supersymmetry breaking and anomaly mediated supersymmetry breaking, the KKLT[4] mechanism gives another mechanism for supersymmetry breaking. This mechanism, mirage supersymmetry breaking, is a mix of moduli stabilization and anomaly mediation. Within the KKLT mechanism there is no difference between mirage and anomaly mediated mechanisms. The pattern of gaugino masses will be able to distinguish different supersymmetry breaking mechanisms.

How to obtain supersymmetric standard model from compactification of $E_{8} \times E_{8}$ heterotic string theory was discussed by B. Ovrut. The mechanism requires breaking $E_{8}$ first to $S O(10)$ and then to $S U(3) \times S U(2) \times U(1)_{Y} \times U(1)_{B-l}$ using $Z_{3} \times Z_{3}$ Wilson lines. This gives rise to 6 geometric and 13 gauge bundle moduli. It does lead to right minimal supersymmetric standard model matter content with Higgs mass at low scale. The model suppresses proton decay and get hierarchical Yukawa couplings. The doublet-triplet splitting projects out triplet Higgs from the model. This construction requires new mathematics like slope stable bundles and Leray spectral sequences.

\section{Phenomenology}

Black holes at colliders were discussed by S. C. Park[5]. It was shown that at colliders, one will generically get rotating black holes due collisions taking place at finite impact parameter. Therefore, all the computations done earlier for non-rotating black holes at the collider do not work. Those black holes whose Schwarzschild radius is smaller than the radius of compactification are intrinsically higher dimensional black holes. These black holes generically Hawking radiate. Rotation of the black hole favors emission of vector particle over fermions and scalars. It is therefore easy to deduce existence of a rotating black hole if we look for hard multigluon events at LHC. Running neutrino masses from six dimensions was discussed by S. Vempati. The active neutrinos are localized on a three-brane and interact with the bulk six dimensional sterile neutrino by brane localized mass term. The renormalization group running of the neutrino mass above compactification scale comes purely from classical effects. This may lead to detectable contribution to neutrinoless beta decay.

\section{References}

[1] N. Sakai, M. Eto, Y. Isozumi, M. Nitta and K. Ohashi, arXiv:hep-th/0703136.

[2] J. M. Cline, arXiv:0705.2982 [hep-th].

[3] K. Choi, arXiv:0705.3330 [hep-th]. 
[4] S. Kachru, R. Kallosh, A. Linde and S. P. Trivedi, Phys. Rev. D 68 (2003) 046005 [arXiv:hep-th/0301240].

[5] S. C. Park, arXiv:0704.1056 [hep-ph]. 